in 2009, accounting for a sevenfold increase since 1999. Accurate treponemal and non-treponemal serological testing are critical to providing a correct diagnosis, but there are substantial variations in the quality of serological diagnostic tests and testing across different parts of China. Since 2003, the Guangdong Provincial STI Control Center in collaboration with the Bureau of Public Health has developed a program on STIs laboratory construction and quality assurance system. We report the proficiency of serological test of syphilis among the STIs laboratories in Guangdong Province in 2004-2009.

Methods Proficiency panels consisting of five samples with syphilis positive and negative sera were prepared in the provincial laboratory and sent to STIs laboratories for non-treponemal and treponemal testing once a year. Participating laboratories were asked to report the type of test used and quantitative and qualitative results for each serum. Each quantitative result was compared to the geometric mean of all participants' results, and was considered correct if it was less than fourfold difference from the mean titre.

Results The numbers of STIs laboratories in Guangdong which participated in the survey increased from 19 to 225 and a total of 13203 sera were tested from 2004 to 2009. 98\% laboratories used the "toluidine red unheated serum" (TRUST) as their non-treponemal test and all laboratories used the "Treponema pallidum particle agglutination assay" (TPPA) as their treponemal test. The mean accuracies of non-treponemal tests among the laboratories evaluated increased significantly from $78.9 \%$ in 2004 to $97.7 \%$ in 2009 $\left(\chi^{2}=17.11, \mathrm{p}<0.01\right)$ for qualitative test results, and from $75.8 \%$ in 2004 to $90.8 \%$ in $2009\left(\chi^{2}=8.09, p<0.01\right)$ for quantitative test results. Higher accuracies were observed with TPPA ranging from 93.5 to $100 \%\left(\chi^{2}=2.85, \mathrm{p}>0.05\right)$ for qualitative test results and improved from $73.7 \%$ to $86.5 \%\left(\chi^{2}=4.7, p<0.05\right)$ for quantitative test results during this period. For non-treponemal testing, $4.1 \%$ $(113 / 2754)$ of the results were found to be false negative and $2.3 \%$ $(34 / 1456)$ were found to be false positive. For qualitative treponemal results, $6.5 \%(96 / 1470)$ and $1.9 \%(17 / 889)$ of treponemal results were found to be false-negative and false-positive respectively. For quantitative results, it was worth noting that in some cases, four to seven-titre deviations were observed for the same sera.

Conclusion This province-wide STIs laboratory construction and quality assurance system has helped to improve the accuracies of serological syphilis testing over time. But there is still room for improvement to facilitate improved control of syphilis in China.

\section{P1-S4.28 SURVEY OF METHODOLOGY USED FOR THE IDENTIFICATION AND ANTIMICROBIAL SUSCEPTIBILITY TESTING OF NEISSERIA GONORRHOEAE IN LATIN AMERICA AND THE CARIBBEAN}

doi:10.1136/sextrans-2011-050108.172

S Starnino, M Liao, M Ruben, A Storey, J A R Dillon, GASP-LAC Network*. Vaccine and Infectious Disease Organization, University of Saskatchewan, Saskatoon, Canada

Background The Gonococcal Antimicrobial Susceptibility Surveillance Program in Latin America and the Caribbean (GASP-LAC) in the 1990s had significant impact in identifying trends in antimicrobial susceptibility in the region. To revitalise the GASP-LAC, a survey was undertaken to determine the level of surveillance activity and the methods used for the identification and antimicrobial susceptibility testing (AST) of Neisseria gonorrhoeae (Ng) isolates.

Methods A structured questionnaire was distributed to potential participants to collect information regarding surveillance activities and methods used for identification and AST of $\mathrm{Ng}$ in LAC countries. Information was also obtained from presentations at the Workshop of the GASP-LAC in November 2010 in Buenos Aires, Argentina.
Results 7 countries completed the questionnaire and four provided unstructured answers regarding the methodologies. All 11 countries were interested in continuing to participate in the GASP-LAC. Of nine countries reporting, seven had an on-going country-wide network for gonococcal AST and two countries collected isolates locally, the number of isolates tested each year varied (25-400). Thayer Martin medium was used for $\mathrm{Ng}$ primary culture by all countries answering this question $(n=8)$; among them, four countries used biochemical tests alone, or coupled with Gram stain $(n=3)$ and one country, in addition to the two methods, used antigen detection and the nucleic acid amplification method. Chromogenic cephalosporin was used by all respondents $(n=9)$ for detecting $B$ lactamase production. Methods used for AST included agar dilution in 6 of 9 reporting countries, coupled with disc diffusion $(n=4)$ and Etest $(n=2)$; the remaining used disc diffusion alone $(n=1)$ or coupled with Etest $(n=2)$. CLSI interpretation criteria were used in all responding participants $(\mathrm{n}=9)$. $\mathrm{Ng}$ reference strains included ATCC49226 $(n=6)$, coupled with WHO III, V, VII $(n=2)$ or WHO A$\mathrm{E}(\mathrm{n}=1)$.

Conclusions Different levels of surveillance were noted between countries probably due to various resource availabilities. On the basis of these responses the GASP-LAC Co-ordinating Centre will reestablish and consolidate the GASP-LAC.

*Authors contributed equally and are listed in an alphabetical order of country names. P Galarza, I Pagano, M E Trigoso, A Schwartz Benzaken, V M Pinto, A Maldonado Ballesteros, 0 M. Sanabria Cruz, A Llop, E Aguilar Jarrin, N Aguayo, J L Portilla Carbajal, G Borthagaray, A Acevedo, D Payares

\section{P1-S4.29 SYNTHESIS OF EVIDENCE ON IMPLEMENTATION RESEARCH ON POINT-OF-CARE SYPHILIS TESTS: A SYSTEMATIC REVIEW}

doi:10.1136/sextrans-2011-050108.173

${ }^{1} \mathrm{Y}$ Jafari, ${ }^{2} \mathrm{M}$ Johri, ${ }^{3} \mathrm{D}$ Ako-Arrey, ${ }^{1} \mathrm{~S}$ Shivkumar, ${ }^{4} \mathrm{G}$ Lambert, ${ }^{5} \mathrm{C}$ Claessens, ${ }^{1} \mathrm{M}$ Klein, ${ }^{6} \mathrm{~J}$ Cajas, ${ }^{7} \mathrm{R}$ Peeling, ${ }^{1} \mathrm{~N}$ Pai. ${ }^{1}$ McGill University, Montreal, Canada; ${ }^{2}$ Université de Montreal, Canada; ${ }^{3}$ University of Saskatchewan, Canada; ${ }^{4}$ Institut national de santé publique du Québec, Canada; ${ }^{5}$ Laboratoire de santé publique du Québec, INSPO, Canada; ${ }^{6}$ Queen's University, Canada; ' London School of Hygiene \& Tropical Medicine, UK

Background With the increase in global prevalence of syphilis, synthesis of evidence of point-of-care (POC) assays is warranted. While a clear methodology exists to meta-analyse diagnostic performance, a clear rubric that incorporates implementation research outcomes (IRO) relevant for policy making is lacking. Recently, Grading of Recommendations Assessment, Development and Evaluation (GRADE) working group called for a shift to emphasis on patient-centred outcomes for making policy recommendations. However, a lack of clarity in defining, elucidating, and reporting of these outcomes prevents their utilisation in practice. Within this context, we reviewed global evidence on IROs for syphilis POC tests.

Method We systematically searched nine electronic databases for the period of January 1980 to September 2010. Articles that addressed IRO regarding POC syphilis tests were reviewed and data extracted. A second reviewer independently reviewed a subset of the articles. Outcomes were synthesised into a narrative review.

Results 31 (48\%) from 64 full text articles assessed were included in the narrative review. Twenty-four studies were cross-sectional, six were case-control, while one was a clustered randomised control trial (RCT). IROs were categorised into: Acceptability, Preference, Feasibility, Prevalence, Barriers and Challenges, and Economic Evaluations of POC tests. Three papers reported outcomes on acceptability, four on preference, ten on feasibility, seven on impact, six on prevalence, seven on barriers and challenges, and seven on economic 


\section{LETTER}

Unusual increase in reported HIV/ AIDS cases among older persons in western Hunan province, China

An unusual increase in HIV/AIDS cases among older people was reported to the Hunan Centers for Disease Control between 2005 and 2007. Cases originated in four rural, western districts of this inland province of China. Given the historical concern for outbreaks of HIV in rural areas due to blood donation, ${ }^{1}$ these cases prompted closer examination to understand the reasons for their appearance and to take measures to prevent further spread.

Eighty cases met our investigation criterion of 50 years or older and underwent a structured interview. The median age was 65 years (range $51-82$ ); $42 \%$ were female. Most were ethnic minorities, $76 \%$ Tujia and $9 \%$ Miao, with low education. Nearly all had been married; 43\% were widowed. Most (54\%) spent time away from their spouse (median >5 years); $10 \%$ were currently sexually active with a spouse; few ever used condoms with their spouse.

Investigation of the possible modes of HIV acquisition suggests most infections among men were from female sex workers (83\% paid for sex, two-thirds in the last 5 years), and among women through infected husbands. One case had a history of selling blood, most recently in 1981. Eight received a blood transfusion, three before 1976 and five after 1984. All denied drug use; all men denied male-male sex; all women denied extramarital sex. Among men buying sex, $97 \%$ never used condoms.

Two-thirds had never heard of HIV prior to their diagnosis. Few (14\%) knew HIV could be transmitted sexually, through blood $(11 \%)$ or from mother to child $(4 \%)$. Of the men reporting commercial sex contact, $82 \%$ had no knowledge that condoms could prevent HIV. Most cases $(86 \%)$ were detected incidentally during the course of treatment for other diseases or because their spouse was HIV-positive. By interview, $78 \%$ indicated their spouse had tested for HIV, of whom $69 \%$ were reported to be positive.

Our investigation highlights that basic information on HIV/AIDS is not reaching all parts of China, and may especially lag among rural and older people. Discussion of sex with older people has been taboo in China, presenting special challenges in finding effective ways to reach them. As treatment extends survival, the cohort of persons living with HIV will also age. The movement of people between urban and rural areas, an ageing population and the shift of the HIV/AIDS epidemic to sexual transmission ${ }^{2}$ are three trends in China that may now have a dangerous intersection.

Acknowledgements We thank Dr Willi McFarland for his editorial assistance. Xi Chen, ${ }^{1}$ Jun Zheng, ${ }^{1}$ Jian Mei He, ${ }^{1}$ BiYun Qin, ${ }^{1}$
Yifei Hu, ${ }^{2}$ Lu Wang, ${ }^{2}$ Ning Wang ${ }^{2}$

${ }^{1}$ AIDS/STI Division, Hunan Provincial CDC, Changsha, China; ${ }^{2}$ The Office of State Council AIDS Working committee(SCAWCO), National Center for HIV/AIDS

Control and Prevention/China CDC, Beijing, China

Correspondence to Dr Yifei Hu, China National Center for HIV/AIDS Control and Prevention/China CDC, No.27 Nanwei Rd, Xuanwu District, Beijing, China; huyifei@yahoo.com

Funding This work was funded by the China National Mega-project of Science Research No.

2008ZX10001-003.

Competing interests None.

Patient consent Obtained.

Ethics approval This study was approved by NCAIDS/China CDC (FWA00001501).

Contributors $\mathrm{XC}$ oversaw and coordinated the fieldwork. JZ, JMH and BYO conducted the fieldwork. $\mathrm{YH}$ completed the survey design, data analysis and drafting of the manuscript. LW and NW provided technical support during and prior to the survey and mobilised some funds to complete the survey.

Provenance and peer review Not commissioned; internally peer reviewed.

Accepted 3 August 2011

Published Online First 26 August 2011

Sex Transm Infect 2011;87:538

doi:10.1136/sextrans-2011-050228

\section{REFERENCES}

1. Wu Z, Liu Z, Detels R. HIV-1 infection in commercial plasma donors in China. Lancet 1995;346:61-2.

2. China Ministry of Health, UNAIDS, and World health Organization (WHO). 2009 Estimates for the HIVIAIDS Epidemic in China. Beijing, China: China Ministry of Health, 2010. http://www.unaids.org.cn/ download/2009\%20China\%20Estimation\%20Report-En. pdf (accessed 1 May 2011).

\section{CORRECTIONS}

doi:10.1136/sextrans-2011-050102.38corr1

RamaKrishnan A. Symposium 9: Applications of program science in the field of STI: S9.3 The programme science of scale: the Avahan experience. Sex Transm Infect 2011;87:A10. doi:10.1136/ sextrans-2011-050102.38.

The author list for this abstract should read: RamaKrishnan A, Sgaier S. doi:10.1136/sextrans-2011-050108.527corr1

Patel S, Pond M. P4-S4.02: A 22-Organism Microarray Approach for Detecting Microbiological Associations with Symptomatic Urethritis in Males. Sex Trans Infect 2011;87:A316. doi:10.1136/ sextrans-2011-050108.527.

The author list for this abstract should read:

Pond $\mathrm{MJ}^{1}$, Patel $\mathrm{S}^{2}$, Hinds $\mathrm{J}^{1}$, Newton $\mathrm{R}^{3}$, Wernisch $\mathrm{L}^{3}$, Butcher $\mathrm{PD}^{1}$ and Sadiq $\mathrm{ST}^{1,2}$

1. Centre for Infection and Immunity, Division of Clinical Sciences, St George's, University of London, UK

2. Department of Genitourinary Medicine, St George's Healthcare NHS Trust, London, UK

3. MRC Biostatistics Unit, Institute of Public Health, Cambridge, UK

A pan-pathogen microarray for detection of microbiological associations with symptomatic urethritis in males.

doi:10.1136/sextrans-2011-050108.45-050108.172corr

Starnino S, Liao M, Ruben M, Storey A, Dillon JAR, GASP-LAC Network. P1-S1.45 Neisseria Gonorrhoeae Antimicrobial Susceptibility in Latin America and the Caribbean (2000-2009) - A Contribution to the Treatment Guidelines Revision. Sex Transm Infect 2011;87:A117. doi:10.1136/ sextrans-2011-050108.45.

Starnino S, Liao M, Ruben M, Storey A, Dillon JAR, GASP-LAC Network. P1S4.28 Survey Of Methodology Used For The Identification And Antimicrobial Susceptibility Testing of Neisseria Gonorrhoeae In Latin America And The Caribbean. Sex Transm Infect 2011;87:A172. doi:10.1136/sextrans-2011050108.172.

The author lists for these abstracts should read: Starnino S, GASP-LAC Network, Liao M, Ruben M, Storey A, Dillon JAR.

doi:10.1136/sextrans-2011-050109.147corr1

Campbell LA, Zidal SV, Patton D, Chochou Kuo. O4-S2.03: An Anti-Adhesive Approach to Prevention of $C$ Trachomatis Infection. Sex Trans Infect 2011;87:A86. doi:10.1136/sextrans-2011-050109.147.

The author's name Zidal SV should be correctly spelt as S Zarate Vidal. 